\title{
Existentially Closed BIBD Block-Intersection Graphs
}

\author{
Neil A. McKay ${ }^{* \dagger}$ and David A. Pike ${ }^{\ddagger}$ \\ Department of Mathematics and Statistics \\ Memorial University of Newfoundland \\ St. John's, Newfoundland \\ Canada. A1C 5S7
}

Submitted: Mar 20, 2007; Accepted: Sep 27, 2007; Published: Oct 16, 2007

Mathematics Subject Classification: 05B05, 05C62

\begin{abstract}
A graph $G$ with vertex set $V$ is said to be $n$-existentially closed if, for every $S \subset V$ with $|S|=n$ and every $T \subseteq S$, there exists a vertex $x \in V-S$ such that $x$ is adjacent to each vertex of $T$ but is adjacent to no vertex of $S-T$. Given a combinatorial design $\mathcal{D}$ with block set $\mathcal{B}$, its block-intersection graph $G_{\mathcal{D}}$ is the graph having vertex set $\mathcal{B}$ such that two vertices $b_{1}$ and $b_{2}$ are adjacent if and only if $b_{1}$ and $b_{2}$ have non-empty intersection.

In this paper we study balanced incomplete block designs (BIBDs) and when their block-intersection graphs are $n$-existentially closed. We characterise the BIBDs with block size $k \geqslant 3$ and index $\lambda=1$ that have 2-e.c. block-intersection graphs and establish bounds on the parameters of BIBDs with index $\lambda=1$ that are $n$-e.c. where $n \geqslant 3$. For $\lambda \geqslant 2$ and $n \geqslant 2$, we prove that only simple $\lambda$-fold designs can have $n$-e.c. block-intersection graphs. In the case of $\lambda$-fold triple systems we show that $n \geqslant 3$ is impossible, and we determine which 2-fold triple systems (i.e., BIBDs with $k=3$ and $\lambda=2$ ) have 2-e.c. block-intersection graphs.
\end{abstract}

Keywords: block designs; block-intersection graphs; existentially closed graphs

\section{Introduction}

Erdős and Rényi first introduced the concept of $n$-existentially closed graphs when considering random graphs [2]. Specifically, a graph $G$ with vertex set $V$ is $n$-existentially

\footnotetext{
*Email: nmckay@mathstat.dal.ca

${ }^{\dagger}$ Current Address: Department of Mathematics and Statistics, Dalhousie University, Halifax, Nova Scotia, Canada, B3H 3J5

${ }^{\ddagger}$ Email: dapike@math.mun.ca

${ }^{\S}$ Corresponding author
} 
closed (or $n$-e.c.) if, for each proper subset $S$ of $V$ with cardinality $|S|=n$ and each subset $T$ of $S$, there exists some vertex $x$ not in $S$ that is adjacent to each vertex of $T$ but to none of the vertices of $S-T$. Hence a graph $G$ is 1 -e.c. if and only if no vertex of $G$ has degree 0 or $(|V|-1)$. A survey paper on the topic of existentially closed graphs appears in [1].

A balanced incomplete block design of order $v$, having block size $k$ and index $\lambda$, or $\operatorname{BIBD}(v, k, \lambda)$, is an ordered pair $(V, \mathcal{B})$, where $V$ is a set of $v$ points and $\mathcal{B}$ is a collection of $k$-subsets of $V$ known as blocks such that every pair of points of $V$ occurs in exactly $\lambda$ blocks of $\mathcal{B}$. The block-intersection graph of such a design $\mathcal{D}$ is the graph $G_{\mathcal{D}}$ having vertex set $\mathcal{B}$, and in which two vertices are adjacent if and only if their corresponding blocks share at least one point of $V$. For further information on combinatorial designs, the reader is referred to [7].

In [3], Forbes, Grannell and Griggs studied the block-interesection graphs of Steiner triple systems; a Steiner triple system of order $v$, or $\operatorname{STS}(v)$, is just a BIBD with parameters $(v, 3,1)$. In particular, they considered when Steiner triple systems have $n$ existentially closed block-intersection graphs. For $n=2$ they concluded that a STS $(v)$ has a 2-e.c. block-intersection graph if and only if $v \geqslant 13$. For $n=3$ they determined that for a $\operatorname{STS}(v)$ to have a 3-e.c. block-intersection graph $v$ must be either 19 or 21 . Two STS(19) with 3-e.c. block-intersection graphs were presented, while the existence of a STS(21) with a 3-e.c. block-intersection graph remained unsettled.

In this present paper we expand on the work of Forbes et al. by considering the more general setting of balanced incomplete block designs. We characterise those $\operatorname{BIBD}(v, k, 1)$ with $k \geqslant 3$ that have 2-e.c. block-intersection graphs, and for $n \geqslant 3$ we obtain bounds on the design parameters for those $\operatorname{BIBD}(v, k, 1)$ for which the block-intersection graph

is $n$-e.c. When $\lambda \geqslant 2$, we obtain similar parameter bounds, and we prove that $\lambda$-fold designs with $n$-e.c. block-intersection graphs are necessarily simple. Further, we determine all $\operatorname{BIBD}(v, 3,2)$ which have 2-e.c. block-intersection graphs, noting that there are no $\operatorname{BIBD}(v, 3,2)$ with $n$-e.c. block-intersection graphs for any $n \geqslant 3$.

\section{A Few Preliminaries}

We begin by observing the following result, the proof of which is trivial.

Lemma 1 If a graph $G$ is n-e.c. and $n>1$, then $G$ is also $(n-1)$-e.c.

Another simple result pertaining to block-intersection graphs is:

Lemma 2 If $\mathcal{D}=(V, \mathcal{B})$ is a $B I B D(v, k, \lambda)$ such that $G_{\mathcal{D}}$ is $n$-e.c., then $v \geqslant(n+1) k$.

Proof. Since $G_{\mathcal{D}}$ is $n$-e.c., then for each $n$-set $S \subset \mathcal{B}$ by selecting $T=\emptyset$ we find that there must exist a vertex $x$ that is adjacent to no vertex of $S$. Inductively, and by using Lemma 1, we conclude that $G_{\mathcal{D}}$ must contain a set of $(n+1)$ independent vertices. Equivalently, the design must contain a set of $(n+1)$ pairwise non-intersecting blocks, each containing $k$ points. Hence $v \geqslant(n+1) k$. 
Throughout the remainder of this paper we adopt the convention that if $\mathcal{D}=(V, \mathcal{B})$ is a $\operatorname{BIBD}(v, k, \lambda)$, then $V=\{1,2, \ldots, v\}$.

We now show that the existential closure of any BIBD is bounded by the block size of the design:

Theorem 1 If the block-intersection graph of a $\operatorname{BIBD}(v, k, \lambda)$ is $n$-e.c., then $n \leqslant k$.

Proof. Suppose that $\mathcal{D}$ is a $\operatorname{BIBD}(v, k, \lambda)$ for which $G_{\mathcal{D}}$ is $n$-e.c. Without loss of generality, we may assume that $\mathcal{D}$ contains the block $b=\{1,2, \ldots, k\}$.

First consider the case in which $k<v$. For each $i=1,2, \ldots, k$, let $b_{i}$ be a block of $\mathcal{D}$ that contains both point $i$ as well as point $v$, and let $B=\left\{b_{1}, b_{2}, \ldots, b_{k}\right\}$ (when $\lambda \geqslant 2$, it may be that $b_{1}, b_{2}, \ldots, b_{k}$ are not all distinct, in which case $\left.|B|<k\right)$. Observe that there is no block $x$ that intersects $b$ but none of $b_{1}, b_{2}, \ldots, b_{k}$. Hence in $G_{\mathcal{D}}$ there can be no vertex $x$ that is adjacent to each vertex of $T=\{b\}$ but no vertex of $S-T$ where $S=T \cup B$. Thus $G_{\mathcal{D}}$ is not $(|B|+1)$-e.c., and by Lemma 1 it follows that $n \ngtr|B|+1$. Hence $n \leqslant|B| \leqslant k$.

In the case where $k=v$, we find that the design $\mathcal{D}$ consists of $\lambda$ copies of a single block, so that $G_{\mathcal{D}}$ is just $K_{\lambda}$. Hence the hypothesis that $G_{\mathcal{D}}$ is $n$-e.c. fails to be satisfied, regardless of the value of $n$.

\section{Designs with Index $\lambda=1$}

In this section we consider designs having index $\lambda=1$. We begin by considering the case of $n=2$, for which we obtain a characterisation for BIBDs with $n$-e.c. block-intersection graphs:

Theorem 2 The block-intersection graph of a $B I B D(v, k, 1)$ with $k \geqslant 3$ is 2-existentially closed if and only if $v \geqslant k^{2}+k-1$.

Proof. For any $\operatorname{BIBD}(v, k, 1) \mathcal{D}=(V, \mathcal{B})$, the replication number $r$ of any point of $V$ is $r=\frac{v-1}{k-1}$. Hence $r \geqslant k+2$ if and only if $v \geqslant k^{2}+k-1$.

To prove the forward implication, let $\mathcal{D}$ be a $\operatorname{BIBD}(v, k, 1)$ such that $k \geqslant 3$ and $G_{\mathcal{D}}$ is 2-e.c. Then the design must contain a pair of disjoint blocks, so without loss of generality let $b_{1}=\{1,2, \ldots, k\}$ and $b_{2}=\{k+1, k+2, \ldots, 2 k\}$ be disjoint elements of $\mathcal{B}$.

Now consider the 2 -subset $S=\left\{b_{1}, b_{2}\right\}$ of $\mathcal{B}$ and the subset $T=\left\{b_{1}\right\}$ of $S$. Since $G_{\mathcal{D}}$ is 2-e.c., then there must exist a third block, say $b_{3}$, such that $\left|b_{1} \cap b_{3}\right|=1$ but $b_{2} \cap b_{3}=\emptyset$. Without loss of generality, let $b_{3}=\{1,2 k+1,2 k+2, \ldots, 3 k-1\}$.

For each $i \in b_{2}$ consider the unique block, say $b_{i}$, that contains the pair $\{1, i\}$. Note that point 1 is now seen to occur in at least $k+2$ blocks (viz. $b_{1}, b_{3}$, and the blocks $\left.b_{k+1}, \ldots, b_{2 k}\right)$ and that point 1 is the only point shared by any pair of these blocks. Hence $r \geqslant k+2$.

We now prove the converse implication. Let $\mathcal{D}$ be a $\operatorname{BIBD}(v, k, 1)$ with $k \geqslant 3$ such that $v \geqslant k^{2}+k-1$. Let $b_{1}$ and $b_{2}$ be two distinct blocks of $\mathcal{B}$ and let $S=\left\{b_{1}, b_{2}\right\}$. For 
each subset $T$ of $S$, we must show that there exists a vertex $x$ of $\mathcal{B}-S$ that is adjacent in $G_{\mathcal{D}}$ to each vertex of $T$ but to no vertex of $S-T$.

Case 1. $\left|b_{1} \cap b_{2}\right|=1$

Without loss of generality, let $b_{1}=\{1,2, \ldots, k\}$ and $b_{2}=\{k, k+1, \ldots, 2 k-1\}$.

If $T=S$, then let $x$ be the unique block that contains the pair $\{1, k+1\}$.

If $|T|=1$, then without loss of generality assume that $T=\left\{b_{1}\right\}$. For each $i \in$ $\{k+1, k+2, \ldots, 2 k-1\}$, let $b_{i}$ be the unique block that contains the pair $\{1, i\}$. The $k$ points of $b_{2}$ are therefore paired with point 1 in the $k$ blocks $b_{1}, b_{k+1}, b_{k+2}, \ldots, b_{2 k-1}$. Recall that $r \geqslant k+2$ and so there exists a block $x$ not in $S$ that contains point 1 but none of the points of $b_{2}$.

If $T=\emptyset$, then let $\nu(t, k-t)$ denote the number of blocks of $\mathcal{B}$ having exactly $t$ points from the set $W=\{1,2, \ldots, 2 k-1\}$ and exactly $k-t$ points from the set $V-W$. Trivially we obtain $\nu(k, 0)=2, \nu(t, k-t)=0$ for each $t \in\{k-1, k-2, \ldots, 3\}$, and $\nu(2, k-2)=(k-1)^{2}$.

Of the $(2 k-1)(v-(2 k-1))$ pairs of points formed by selecting one point from $W$ and another from $V-W$, each of the $(k-1)^{2}$ blocks enumerated by $\nu(2, k-2)$ contains $2(k-2)$ of them. Thus $\nu(1, k-1)=\frac{(2 k-1)(v-(2 k-1))-2(k-2)(k-1)^{2}}{k-1}$.

Since $|\mathcal{B}|=\frac{v(v-1)}{k(k-1)}$ in any $\operatorname{BIBD}(v, k, 1)$, it now follows that $\nu(0, k)=\frac{v(v-1)}{k(k-1)}-\nu(k, 0)-$ $\nu(2, k-2)-\nu(1, k-1)=\frac{v^{2}-v+k^{2}+k^{4}-k^{3}-2 k^{2} v+k v}{k(k-1)}$. We need to establish that $\nu(0, k) \geqslant 1$, but since $\nu(0, k)$ is an integer and $k \geqslant 3$, it is sufficient to prove that $N=\left(v^{2}-v+k^{2}+\right.$ $\left.k^{4}-k^{3}-2 k^{2} v+k v\right)$ is positive. Recalling that $v \geqslant k^{2}+k-1$, let $\epsilon=v-\left(k^{2}+k-1\right) \geqslant 0$ so that we now have $N=\left(2 k^{2}-4 k+3 k \epsilon+\epsilon^{2}-3 \epsilon+2\right)$. Clearly $2 k^{2}>4 k$ and $3 k \epsilon \geqslant 3 \epsilon$ for all $k \geqslant 3$, and thus $N>0$.

Case 2. $\left|b_{1} \cap b_{2}\right|=0$

Without loss of generality, let $b_{1}=\{1,2, \ldots, k\}$ and $b_{2}=\{k+1, \ldots, 2 k\}$.

If $T=S$, then let $x$ be the unique block that contains the pair $\{1, k+1\}$.

If $|T|=1$, then without loss of generality assume that $T=\left\{b_{1}\right\}$. For each $i \in b_{2}$, let $b_{i}$ be the unique block that contains the pair $\{1, i\}$. The $k$ points of $b_{2}$ are therefore paired with point 1 in the $k$ blocks $b_{k+1}, b_{k+2}, \ldots, b_{2 k}$. Recall that $r \geqslant k+2$ and so there exists a block $x \neq b_{1}$ that contains point 1 but none of the points of $b_{2}$.

If $T=\emptyset$, then let $\nu(t, k-t)$ denote the number of blocks of $\mathcal{B}$ having exactly $t$ points from the set $W=\{1,2, \ldots, 2 k\}$ and exactly $k-t$ points from the set $V-W$. Trivially we obtain $\nu(k, 0)=2, \nu(t, k-t)=0$ for each $t \in\{k-1, k-2, \ldots, 3\}$, and $\nu(2, k-2)=k^{2}$. It follows that $\nu(1, k-1)=\frac{2 k(v-2 k)-2(k-2)\left(k^{2}\right)}{k-1}$ and hence $\nu(0, k)=\frac{v(v-1)}{k(k-1)}-\nu(k, 0)-\nu(2, k-$ $2)-\nu(1, k-1)=\frac{v^{2}-v-2 k^{2}+2 k+k^{4}+k^{3}-2 k^{2} v}{k(k-1)}$. Let $N=\left(v^{2}-v-2 k^{2}+2 k+k^{4}+k^{3}-2 k^{2} v\right)$ and let $\epsilon=v-\left(k^{2}+k-1\right) \geqslant 0$. Then $N=\left(k^{3}-2 k^{2}-k+2 k \epsilon+\epsilon^{2}-3 \epsilon+2\right)$ is positive since $\left(k^{3}-2 k^{2}-k\right)>0$ and $2 k \epsilon \geqslant 3 \epsilon$ whenever $k \geqslant 3$.

We now obtain Theorem 3.1 of [3] as a corollary:

Corollary 1 The block-intersection graph of a STS $(v)$ is 2-e.c. if and only if $v \geqslant 13$. 
Proof. Theorem 2 asserts that the block-intersection graph of a STS $(v)$ is 2-e.c. if and only if $v \geqslant 11$. However, a Steiner triple system of order $v$ exists if and only if $v \equiv 1$ or 3 (mod 6), thereby eliminating orders 11 and 12 from consideration.

We now begin to consider those $\operatorname{BIBD}(v, k, 1)$ having $n$-e.c. block-intersection graphs where $n$ exceeds 2 . At this point it is helpful to recall that an independent set of points in a design $\mathcal{D}=(V, \mathcal{B})$ is a subset $I \subset V$ such that no block of $\mathcal{B}$ has all of its points contained within $I$. By $I_{k}(v)$ we denote the maximum cardinality of an independent set in any $\operatorname{BIBD}(v, k, \lambda)$; i.e., the maximum over all $\operatorname{BIBDs}$ with parameters $(v, k, \lambda)$. For triple systems, the value of $I_{3}(v)$ was established by Sauer and Schönheim to be at most $\frac{v+1}{2}[6]$. We now present a modest generalisation of the result by Sauer and Schönheim:

Lemma $3 I_{k}(v) \leqslant \frac{(v-1)(k-2)}{k-1}+1$.

Proof. Let $\mathcal{D}=(V, \mathcal{B})$ be a $\operatorname{BIBD}(v, k, \lambda)$, let $I$ be an independent set of points in $\mathcal{D}$, and let $p \in I$. Note that there are $|I|-1$ pairs of points of the form $\{p, q\}$ where $q$ is a point of $I$ other than $p$, and that each such pair occurs $\lambda$ times. Each block of the design can contain at most $(k-2)$ of these pairs without contradicting the status of $I$ as an independent set. Hence there must be at least $\frac{\lambda(|I|-1)}{k-2}$ blocks that contain pairs of points of this form. Necessarily this quantity cannot exceed the replication number, $r=\frac{\lambda(v-1)}{k-1}$, of the design. Therefore $\frac{\lambda(|I|-1)}{k-2} \leqslant \frac{\lambda(v-1)}{k-1}$ and the result follows.

We now use Lemma 3 to establish upper bounds on the orders of designs having $n$-e.c. block-intersection graphs.

Theorem 3 Let $n \geqslant 3$. If $\mathcal{D}=(V, \mathcal{B})$ is a $\operatorname{BIBD}(v, k, 1)$ for which the block-intersection graph is $n$-e.c., then $v \leqslant k^{4}-n k^{3}+(2 n-2) k^{2}-n k+k+1$.

Proof. Since $G_{\mathcal{D}}$ is $n$-e.c., then there must exist a set of $(n+1)$ pairwise disjoint blocks in the design, although we do not require that many. Let $D$ be a set of $(n-1)$ pairwise disjoint blocks in $\mathcal{B}$, and let $b_{1}$ and $b_{2}$ be two distinct blocks of $D$.

Let $B \subset \mathcal{B}$ be the set of all $k^{2}$ blocks that contain a point from $b_{1}$ as well as a point from $b_{2}$. Since $\lambda=1$, each block $b \in B$ contains at most one point from each block of $D$, and hence $b$ contains at least $(k-(n-1))$ points that do not occur as points in any of the blocks of $D$ (recall from Theorem 1 that $n \leqslant k$ ). So for each block $b \in B$, let $P_{b}$ be an arbitrary but fixed set of $(k-(n-1))$ points of $b$, none of which occur as a point of any block of $D$. Let $P=\left(\bigcup_{b \in D} b\right) \cup\left(\bigcup_{b \in B} P_{b}\right)$ and observe that $|P| \leqslant k(n-1)+(k-n+1) k^{2}$.

Suppose now that $v>\left(\frac{(v-1)(k-2)}{k-1}+1\right)+\left(k(n-1)+(k-n+1) k^{2}\right)$. Then by Lemma 3 , $v-|P|>I_{k}(v)$, and so there must exist a block, say $b_{n}$, that uses none of the points of $P$. Let $S=D \cup\left\{b_{n}\right\}$ and $T=S$. Since $G_{\mathcal{D}}$ is $n$-e.c., then there must exist a block $x$ that intersects all $n$ blocks of $T$. Since $x$ intersects each of $b_{1}$ and $b_{2}$, then necessarily $x$ must 
be one of the blocks of $B$. Moreover, since $x$ intersects each block of $D$, and each pair of blocks of $D$ has empty intersection, then $x$ contains exactly one point from each of the $(n-1)$ blocks of $D$ and so $x$ contains exactly $(k-(n-1))$ points not found in any block of $D$.

Note that $x$ is also adjacent to $b_{n}$, which shares no points with any block of $D$. Hence $x$ and $b_{n}$ must intersect in one of the $(k-(n-1))$ points of $x$ that are not found in any block of $D$, meaning that $x$ and $b_{n}$ must intersect in a point of $P_{x}$. We therefore have a contradiction, since $b_{n}$ contains none of the points of $P_{x}$. Hence $v \leqslant$ $\left(\frac{(v-1)(k-2)}{k-1}+1\right)+\left(k(n-1)+(k-n+1) k^{2}\right)$.

Theorem 3 has the effect of imposing an upper bound on the possible order $v$ for any $\operatorname{BIBD}(v, k, 1)$ having an $n$-e.c. block-intersection graph. For instance, when coupled with Theorem 2 and Lemma 1, the only possible orders for a $\operatorname{BIBD}(v, 4,1)$ with a 3 -e.c. block-intersection graph are seen to be those in the interval $19 \leqslant v \leqslant 121$, which can be refined to $25 \leqslant v \leqslant 121$ by noting that the existence of a $\operatorname{BIBD}(v, 4,1)$ requires that $v \equiv 1$ or $4(\bmod 12)$.

\section{$4 \lambda$-fold Designs}

We now consider BIBDs in which the index $\lambda$ need not be 1 . When $\lambda \geqslant 2$, it is possible for designs to contain repeated blocks. A design with no repeated blocks is said to be simple, and as is now shown, only for simple designs can the block-intersection graph be $n$-e.c. when $n \geqslant 2$.

Lemma 4 If $\lambda \geqslant 2, n \geqslant 2$, and $\mathcal{D}=(V, \mathcal{B})$ is a $B I B D(v, k, \lambda)$ such that $G_{\mathcal{D}}$ is $n$-e.c., then $\mathcal{D}$ is simple.

Proof. By way of contradiction, suppose that $\mathcal{B}$ contains repeated blocks, say $b_{1}$ and $b_{1}^{\prime}$ (so $\left|b_{1} \cap b_{1}^{\prime}\right|=k$ ). By choosing $S=\left\{b_{1}, b_{1}^{\prime}\right\}$ and $T=\left\{b_{1}\right\}$ we find that there is no vertex $x$ of $G_{\mathcal{D}}$ that is adjacent to $b_{1}$ but not adjacent to $b_{1}^{\prime}$. Hence $G_{\mathcal{D}}$ cannot be 2-e.c., and by Lemma $1, G_{\mathcal{D}}$ cannot be $n$-e.c. for any $n \geqslant 2$. Thus we have the desired contradiction.

Paralleling the situation with 1-fold designs, upper bounds on the orders of designs having $n$-e.c. block-intersection graphs can be obtained for $\lambda$-fold designs:

Theorem 4 Let $n \geqslant 3$. If $\mathcal{D}=(V, \mathcal{B})$ is a $B I B D(v, k, \lambda)$ for which the block-intersection graph is n-e.c., then $v \leqslant \lambda k^{4}-\lambda n k^{3}+(\lambda+1)(n-1) k^{2}-n k+k+1$.

Proof. The proof is similar to that of Theorem 3, except that $|B| \leqslant \lambda k^{2}$, and for each $b \in B$ containing $(k-(n-1))$ or more points not in $D$ we define $P_{b}$ to be a fixed set of $(k-(n-1))$ of these points, whereas for those $b$ having fewer than $(k-(n-1))$ such points we define $P_{b}$ to be all of the points of $b$ which do not appear in $D$. Hence $|P| \leqslant k(n-1)+\lambda k^{2}(k-n+1)$. 
Moreover, when $\lambda \geqslant 2$ we can improve on the bound established by Theorem 1 :

Theorem 5 If $\lambda \geqslant 2$ and $\mathcal{D}=(V, \mathcal{B})$ is a $\operatorname{BIBD}(v, k, \lambda)$ such that $G_{\mathcal{D}}$ is n-e.c., then $n \leqslant\left\lfloor\frac{k+1}{2}\right\rfloor$.

Proof. Suppose that $\mathcal{D}$ is a $\operatorname{BIBD}(v, k, \lambda)$ for which $G_{\mathcal{D}}$ is $n$-e.c. Without loss of generality, we may assume that $\mathcal{B}$ contains the block $b=\{1,2, \ldots, k\}$. Let $T=\{b\}$.

If $k$ is even, then for each $i=1,2, \ldots, \frac{k}{2}$, let $b_{i}$ be a block other than $b$ that contains the two points $(2 i-1)$ and $2 i$. Let $B=\left\{b_{1}, \ldots, b_{\frac{k}{2}}\right\}$ and let $S=T \cup B$. Possibly $b_{i}=b_{j}$ for some $1 \leqslant i<j \leqslant \frac{k}{2}$, in which case $|B|<\frac{k}{2}$.

If $k$ is odd, then for each $i=1,2, \ldots, \frac{k-1}{2}$, let $b_{i}$ be a block other than $b$ that contains the two points $(2 i-1)$ and $2 i$. Let $b_{\frac{k+1}{2}}$ be a block other than $b$ that contains point $k$. Let $S=T \cup\left\{b_{1}, b_{2}, \ldots, b_{\frac{k+1}{2}}\right\}$.

Observe now that in $G_{\mathcal{D}}^{2}$ there is no vertex $x$ that is adjacent to $b$ but to no vertex of $S-T$ and hence $G_{\mathcal{D}}$ is not $|S|$-e.c. It follows from Lemma 1 that $n \leqslant|S|-1 \leqslant\left\lfloor\frac{k+1}{2}\right\rfloor$.

Theorem 5, as it applies to triple systems, states that there are no $\lambda$-fold triple systems with 3-e.c. block-intersection graphs. Accordingly, we now focus on the question of determining which simple $\lambda$-fold triple systems have 2-e.c. block-intersection graphs. We consider in detail the case of $\lambda=2$, beginning by showing that every simple $\operatorname{BIBD}(v, 3,2)$ with $v \geqslant 13$ has a 2-e.c. block-intersection graph.

Lemma 5 If $\mathcal{D}=(V, \mathcal{B})$ is a simple $B I B D(v, 3,2)$ such that $v \geqslant 13$, then $G_{\mathcal{D}}$ is 2-e.c.

Proof. For any $\operatorname{BIBD}(v, k, \lambda)$, the replication number $r$ of any point of $V$ is $r=\frac{\lambda(v-1)}{k-1}$, and so for the design $\mathcal{D}$ we have $r=v-1 \geqslant 12$.

Let $b_{1}$ and $b_{2}$ be two distinct blocks of $\mathcal{B}$ and let $S=\left\{b_{1}, b_{2}\right\}$. For each subset $T$ of $S$, we must show that there exists a vertex $x \in \mathcal{B}-S$ that is adjacent in $G_{\mathcal{D}}$ to each vertex of $T$ but to no vertex of $S-T$.

Case 1. $\left|b_{1} \cap b_{2}\right|=2$

Without loss of generality, let $b_{1}=\{1,2,3\}$ and $b_{2}=\{1,2,4\}$.

If $T=S$, then let $x$ be one of the two blocks that contain the pair $\{3,4\}$.

If $|T|=1$, then without loss of generality assume that $T=\left\{b_{1}\right\}$. Consider the blocks that contain the pairs of points $\{1,3\},\{2,3\}$, and $\{3,4\}$. Since $\lambda=2$ there can be at most six such blocks; in fact there can be at most five, since $b_{1}$ contains an instance of the pair $\{1,3\}$ as well as an instance of the pair $\{2,3\}$ together in a single block. These blocks all contain point 3 , and they each intersect both of $b_{1}$ and $b_{2}$. However, there are at least $(r-5)$ other blocks that also contain point 3 but none of the points of $b_{2}$. Since $r \geqslant 12$, then there exists a vertex $x$ in $G_{\mathcal{D}}$ that is adjacent to $b_{1}$ but is not adjacent to $b_{2}$.

If $T=\emptyset$, then consider the blocks that contain point 5. Since $\left|b_{1} \cup b_{2}\right|=4$ and $\lambda=2$, at most eight blocks of the design will contain point 5 as well as some point from $b_{1} \cup b_{2}$. Since $r \geqslant 12$, then there exist other blocks that contain point 5 but none of the points of $b_{1} \cup b_{2}$; any such block is a suitable choice for $x$. 
Case 2. $\left|b_{1} \cap b_{2}\right|=1$

Without loss of generality, let $b_{1}=\{1,2,3\}$ and $b_{2}=\{1,4,5\}$.

If $T=S$, then let $x$ be one of the two blocks that contain the pair $\{3,4\}$.

If $|T|=1$, then without loss of generality assume that $T=\left\{b_{1}\right\}$. Consider the blocks that contain the pairs of points $\{1,3\},\{3,4\}$, and $\{3,5\}$. Since $\lambda=2$ there can be at most six such blocks. These blocks all contain point 3 , and they each intersect both of $b_{1}$ and $b_{2}$. However, there are at least $r-6 \geqslant 6$ other blocks that also contain point 3 but none of the points of $b_{2}$; any such block is a suitable choice for $x$.

If $T=\emptyset$, then consider the blocks that contain point 6 . Since $\left|b_{1} \cup b_{2}\right|=5$ and $\lambda=2$, at most ten blocks of the design will contain point 6 as well as some point from $b_{1} \cup b_{2}$. Since $r \geqslant 12$, then there exist other blocks that contain point 6 but none of the points of $b_{1} \cup b_{2}$; any such block is a suitable choice for $x$.

Case 3. $\left|b_{1} \cap b_{2}\right|=0$

Without loss of generality, let $b_{1}=\{1,2,3\}$ and $b_{2}=\{4,5,6\}$.

If $T=S$, then let $x$ be one of the two blocks that contain the pair $\{3,4\}$.

If $|T|=1$, then without loss of generality assume that $T=\left\{b_{1}\right\}$. Consider the blocks that contain the pairs of points $\{3,4\},\{3,5\}$, and $\{3,6\}$. Since $\lambda=2$ there can be at most six such blocks. These blocks all contain point 3 , and they each intersect both of $b_{1}$ and $b_{2}$. However, there are at least $r-6 \geqslant 6$ other blocks that also contain point 3 but none of the points of $b_{2}$; any such block is a suitable choice for $x$.

If $T=\emptyset$, then consider the blocks that contain point 7. Since $\left|b_{1} \cup b_{2}\right|=6$ and $\lambda=2$, at most twelve blocks of the design will contain point 7 as well as some point from $b_{1} \cup b_{2}$. If $r>12$, then there exist other blocks that contain point 7 but none of the points of $b_{1} \cup b_{2}$; any such block is a suitable choice for $x$.

However, if $r=12$ then either $G_{\mathcal{D}}$ is 2-e.c. or else each vertex of $G_{\mathcal{D}}$ is a neighbour of at least one of $b_{1}$ and $b_{2}$. We suppose that the latter of these two cases is at hand, in which case each block of $\mathcal{B}$ must contain a point from $b_{1} \cup b_{2}$. In particular, each of the $r=12$ blocks in which point 7 occurs must also contain a point from $b_{1} \cup b_{2}$. Since $\lambda=2$, point 7 is paired with each point of $b_{1} \cup b_{2}$ exactly twice, yielding a total of 12 pairings throughout the design. Hence each of the $r=12$ blocks in which point 7 occurs must contain exactly one pair of points of the form $\{p, 7\}$ where $p \in b_{1} \cup b_{2}$. Consider now the subset $W=\{8,9,10,11,12,13\}$ of $V$. There are $\lambda\left(\begin{array}{c}6 \\ 2\end{array}\right)=30$ pairs of points of the form $\{p, q\}$ where $p, q \in W$, each of which must be in a block with a point of $b_{1} \cup b_{2}$. Hence there are exactly 30 blocks of the form $\{z, p, q\}$ where $z \in b_{1} \cup b_{2}$ and $p, q \in W$. A further 12 blocks are of the form $\{z, 7, p\}$ where $z \in b_{1} \cup b_{2}$ and $p \in W$. None of these 42 blocks contain any of the $\lambda\left(\begin{array}{l}6 \\ 2\end{array}\right)=30$ pairs of points of the form $\{p, q\}$ where $p, q \in b_{1} \cup b_{2}$. Thus the remaining $|\mathcal{B}|-42=10$ blocks (i.e., $b_{1}, b_{2}$, and eight others) contain only points from $b_{1} \cup b_{2}$ and so form a $\operatorname{BIBD}(6,3,2)$. As noted in [4], there is a unique $\operatorname{BIBD}(6,3,2)$. It contains no pair of disjoint blocks such as our $b_{1}$ and $b_{2}$, and so we have the desired contradiction.

Next we eliminate several small orders from possible consideration. From Lemma 2, it follows that if $\mathcal{D}=(V, \mathcal{B})$ is a simple $\operatorname{BIBD}(v, 3,2)$ such that $G_{\mathcal{D}}$ is 2-e.c., then $v \geqslant 9$, so we only need to consider the admissible orders beginning with $v=9$ : 
Lemma 6 No simple $\operatorname{BIBD}(9,3,2)$ has a 2-e.c. block-intersection graph.

Proof. Suppose that $\mathcal{D}=(V, \mathcal{B})$ is a simple $\operatorname{BIBD}(9,3,2)$ such that $G_{\mathcal{D}}$ is 2-e.c. and without loss of generality assume that $b_{1}=\{1,2,3\} \in \mathcal{B}$. Note that $r=8$ and so there are precisely 18 blocks that intersect $b_{1}$, namely the three other blocks containing the pairs $\{1,2\},\{1,3\}$ and $\{2,3\}$ plus $3(r-3)$ blocks that each intersect $b_{1}$ in a single point.

Since $|\mathcal{B}|=24$, then there are exactly five blocks that are disjoint from $b_{1}$. Let $b_{2}$ be a block that is disjoint from $b_{1}$. By selecting $S=\left\{b_{1}, b_{2}\right\}$ and $T=\emptyset$ then it follows

from $G_{\mathcal{D}}$ being 2-e.c. that there must be a third block that is disjoint from both $b_{1}$ and $b_{2}$. Since $v=9$, this third block must be $V-\left(b_{1} \cup b_{2}\right)$. Thus for each block $b_{2}$ that is disjoint from $b_{1}$, there is a corresponding block $b_{2}^{\prime}=V-\left(b_{1} \cup b_{2}\right)$ that is also disjoint from $b_{1}$. Hence the five blocks that are disjoint from $b_{1}$ can be naturally partitioned into pairs, which is a contradiction since 5 is an odd integer.

Lemma 7 No simple $\operatorname{BIBD}(10,3,2)$ has a 2-e.c. block-intersection graph.

Proof. Of the 960 non-isomorphic $\operatorname{BIBD}(10,3,2)$ designs, only 394 are simple. We tested each of these by computer and found that none of them have a 2-e.c. block-intersection graph.

Östergård [5] reported that there are 88616310 non-isomorphic simple $\operatorname{BIBD}(12,3,2)$ designs. We generated these designs (thereby independently confirming their enumeration) and subsequently tested each of their block-intersection graphs to determine which ones are 2-e.c. The vast majority are 2-e.c., whereas as only 286962 fail to be 2-e.c.

We now summarise the status of 2 -fold triple systems with the following theorem:

Theorem 6 If $\mathcal{D}$ is a simple $B I B D(v, 3,2)$ and $G_{\mathcal{D}}$ is 2-e.c., then $v \geqslant 12$. Conversely, the block-intersection graph of any simple $\operatorname{BIBD}(v, 3,2)$ with $v \geqslant 13$ has a 2-e.c. blockintersection graph.

\section{Discussion}

Reflecting on Theorem 1, we pose the following question:

Question 1 For each $k \geqslant 3$, does there exist a $\operatorname{BIBD}(v, k, 1)$ that has a $k$-e.c. blockintersection graph?

This question is answered in the affirmative for $k=3$ as Forbes et al. found two STS(19) designs with 3-e.c. block-intersection graphs [3].

For designs with index $\lambda \geqslant 2$, Theorem 5 suggests that the corresponding question should be:

Question 2 For each $\lambda \geqslant 2$ and $k \geqslant 3$, does there exist a $B I B D(v, k, \lambda)$ for which the block-intersection graph is $\left\lfloor\frac{k+1}{2}\right\rfloor$-e.c.?

In the case where $\lambda=2$ and $k=3$, Theorem 6 establishes an affirmative answer to this question. 


\section{Acknowledgements}

Both authors acknowledge support from NSERC. Thanks are also extended to Brendan McKay for use of his autoson software.

\section{References}

[1] A. Bonato. The search for $n$-e.c. graphs, preprint.

[2] P. Erdős and A. Rényi. Asymmetric graphs, Acta Math. Acad. Sci. Hungar 14 (1963) 295-315.

[3] A.D. Forbes, M.J. Grannell, and T.S. Griggs. Steiner triple systems and existentially closed graphs, Electronic Journal of Combinatorics 12 (2005) \#R42.

[4] R. Mathon and A. Rosa, 2- $(v, k, \lambda)$ Designs of Small Order, The CRC Handbook of Combinatorial Designs, edited by C. Colbourn and J. Dinitz, CRC Press (1996) $3-41$.

[5] P.R.J. Östergård. Enumeration of 2-(12,3,2) designs, Australasian Journal of Combinatorics 22 (2000) 227-231.

[6] N. Sauer and J. Schönheim. Maximal subsets of a given set having no triple in common with a Steiner triple system on the set, Canad. Math. Bulletin 12 (1969) $777-778$.

[7] D.R. Stinson. Combinatorial Designs: Constructions and Analysis. Spinger-Verlag, New York (2004). 